\title{
Influence of Geometric-Confinement Parameters on the Electronic Properties of Quantum Ring
}

\author{
S. Moskal And B.J. SpISAK* \\ Faculty of Physics and Applied Computer Science \\ AGH - University of Science and Technology \\ al. Mickiewicza 30, 30-059 Kraków, Poland
}

(Received June 25, 2007)

\begin{abstract}
We investigate the electronic properties of the quantum rings modelled by a two-dimensional non-singular potential with the cylindrical symmetry as well as the influence of the potential's parameters on energy spectra. In the presence of the external magnetic field we also calculated the persistent currents in such structures in the ballistic regime.
\end{abstract}

PACS numbers: 73.23.Ra, 73.22.Dj, 73.43.Cd

\section{Introduction}

Physical understanding of the electronic properties of nanostructures allows us to fabricate the electronic elements which are widely applied as components of the new generation of electronic and photonic devices. The potential application of such elements in industry stimulates rapid development in nanotechnology and gives us motivation for preparing and studying a new class of confined structures. One of them are quantum rings that are very interesting objects due to potential implementations in optical devices or quantum computing based on a solid state environment. Therefore, the quantum rings have been extensively studied during the last decade both experimentally [1-6] and theoretically [7-10]. Many theoretical studies have investigated various aspects of the one-dimensional quantum rings (for a recent review, see [11]) but the real experiments performed on the semiconductor quantum rings clearly show that these structures possess a finite width in the plane perpendicular to the ring axis [3]. These experimental results allowed one to introduce various theoretical models of confinement potentials for

*corresponding author; e-mail: spisak@novell.ftj.agh.edu.pl 
two-dimensional quantum rings [7, 12-14]. Recently, a few theoretical works have been devoted to the description of a three-dimensional ring $[15,16]$.

At present, quantum rings are prepared by the standard techniques such as optical litography, electron beam litography, or self-organized Stranski-Krastanov growth. The application of self-organized techniques allows one to fabricate the semiconductor quantum rings with complex cross-section profile in the nanoscopic scale [6]. In such systems the number of electrons can be precisely controlled. In real experiments (e.g. Lorke et al. [3]), the self-organized $\operatorname{In}_{x} \mathrm{Ga}_{1-x}$ As quantum rings contain one or two electrons. The shape of quantum ring is determined by co-existence of a few effects: the diffusion, strain and surface/interface energies. These effects can lead to complicated shapes of the confinement potentials. In other words, nontrivial geometry of self-organized quantum ring determines the electronic properties. The usual approach assumes a parabolic confinement potential of the ring with an average radius in the plane of the ring $[7,17]$ but it is far from realistic self-assembled quantum rings for which the confinement potential is originating from the growth process of such structures. We would like to clarify how the electronic properties are affected by the shape of the confinement potential of the quantum ring. Additionally, we consider the influence of an external magnetic field on its electronic properties. One of the particularly interesting effects that are observed in the magnetic field is the persistent current which is regarded as equilibrium properties of the system $[18,19]$. The detection of persistent current in single, isolated [20, 21], or assembly of semiconductor rings [22] is based on the measurements of the magnetic response which oscillates with a fundamental period $h / e$. The amplitude of this oscillatory component corresponds to a persistent current. Recently, a sudden application of persistent currents in the quantum computing was proposed by Zipper et al. [23]. They argue that semiconductor or metal rings with a barrier can lead to a formation of a qubit at low temperatures.

The main purpose of this paper is to explore the influence of the different geometric-confinement parameters and an external magnetic field on the electronic spectrum of semiconductor quantum rings. In the first part of our theoretical investigation, we concentrate on the influence of geometric-confinement parameters of the two-dimensional semiconductor quantum ring on the electronic spectrum. Afterward, we consider the effect of an external magnetic field on the electronic spectrum and we calculate the persistent current for different values of geometric-confinement parameters in the ballistic regime, where the mean free path exceeds the ring size. Our numerical calculations are based on one-band electron Hamiltonian within the effective-mass approximation. In the present study the electron-electron interaction is neglected (although the carrier density is very low) because we are interested in the influence of the geometric parameters of confinement potential on electronic properties of the ring. 


\section{Model of confinement potential}

The potential that confines electrons in quantum rings is difficult to be estimated from the first principles as it was mentioned above. The profile of the confinement potential is not well known for quantum rings and strongly depends on the technique of fabrication $[1,6]$. The experimental data based on scanning tunnelling microscopy or atomic force microscopy (AFM) measurements show that the height of fabricated quantum rings is about $2 \mathrm{~nm}$, whereas an outer diameter in the plane ranges between 30 and $140 \mathrm{~nm}$ and an inner one of about $20 \mathrm{~nm}$ $[2,3,5]$. It implies that confinement along the vertical direction is very strong, while in the plane it is weaker. Therefore, in order to model such systems we use the confinement potential $V_{\text {conf }}$ which possesses the rotational symmetry along the growth axis. In this case we may split the potential $V_{\text {conf }}$ into two components, as follows:

$$
V_{\text {conf }}(r, \varphi, z)=\left\{\begin{array}{l}
V_{\|}(z), \\
V_{\perp}(r, \varphi),
\end{array}\right.
$$

where $V_{\|}(z)$ is the confinement potential along the vertical direction (growth axis). We assume that $V_{\|}(z)$ is modelled by the infinite well potential. The component $V_{\perp}(r, \varphi)$, in the $x-y$ plane is called the lateral potential (see Fig. 1) and we propose it in the form

$$
V_{\perp}(r)=-V_{0}\left[\left(\frac{r}{R_{0}}\right)^{2 p}+b\right] \exp \left(-\left(\frac{r}{R_{1}}\right)^{2 q}\right),
$$

where $V_{0}$ is the depth of the potential and we set it to $200 \mathrm{meV}$. The rest of parameters $R_{0}, R_{1}, b, p$, and $q$ are responsible for the shape of potential as it is shown in Fig. 2. The parameter $V_{0}$ serves as a normalization coefficient and let us set the depth of the lateral potential. Among six parameters mentioned above we concentrate on the influence of four of them: $R_{1}, p, q$, and $b$ as those which significantly change profile of the potential and in consequence the energy

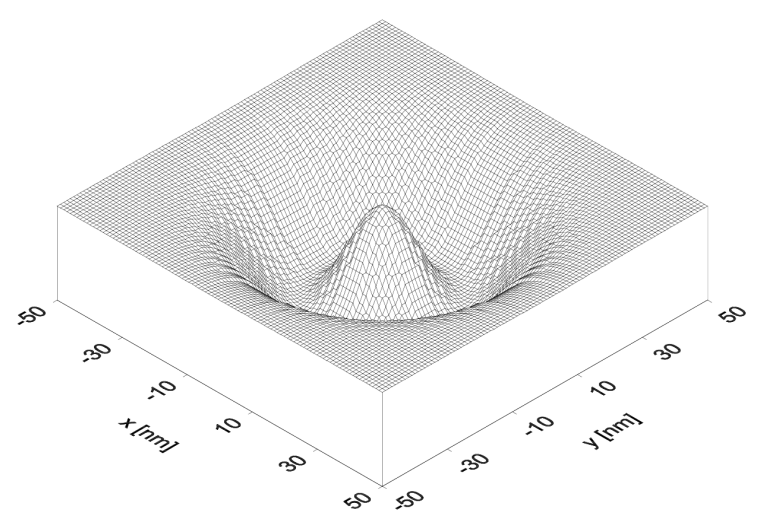

Fig. 1. $2 \mathrm{D}$ profile of the lateral potential for the parameters $V_{0}=200 \mathrm{meV}, R_{1}=$ $18.5 \mathrm{~nm}, b=0, p=1.0$. 

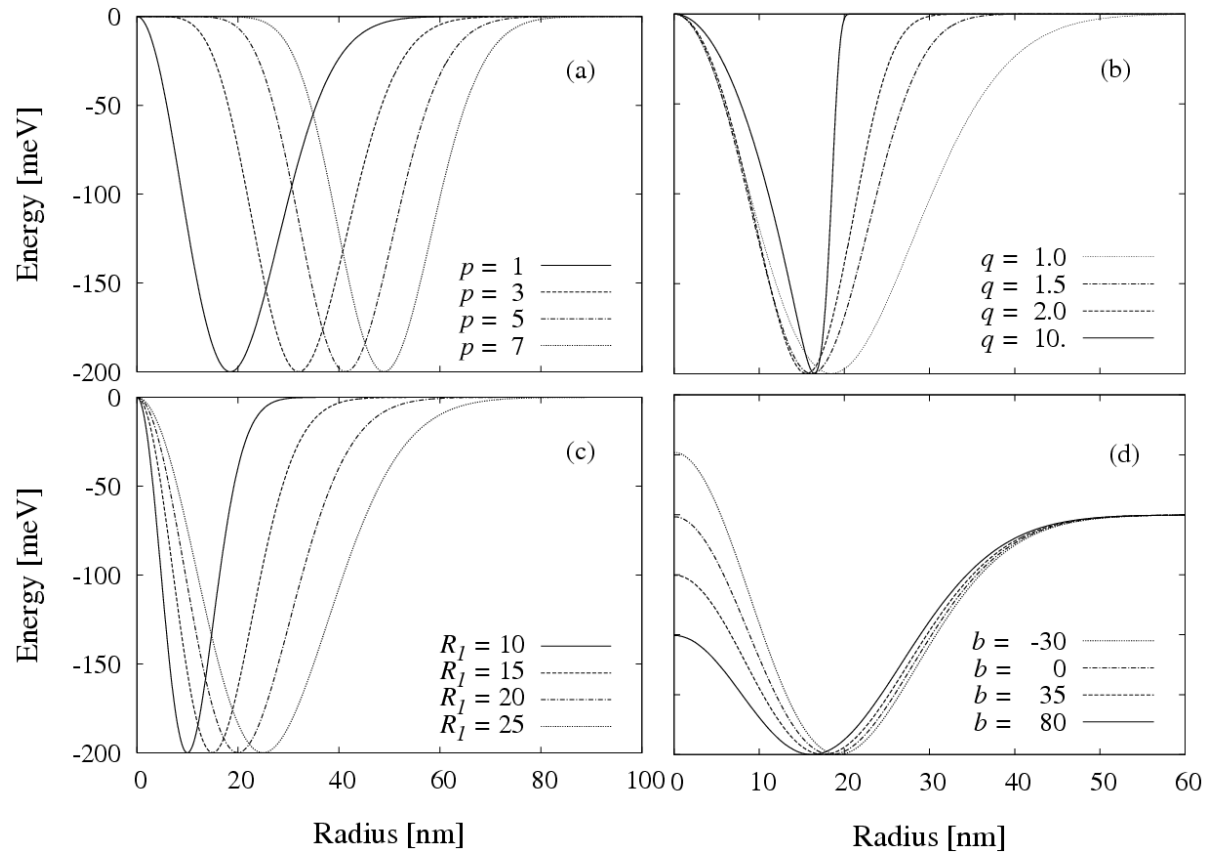

Fig. 2. The influence of key parameters on the shape of the lateral potential.

spectrum. In this case we can neglect the influence of parameter $R_{0}$, because it changes only the depth of the potential and during normalization to the level $V_{0}$ it is cancelled. The first one, $R_{1}$, is responsible for the width of the ring's core. The larger it is the broader becomes the core. The second parameter, $p$, changes the width of the ring's core. When $p$ increases, the broader the core is and simultaneously the core walls become steeper. The third parameter, $q$, changes the inclination of outer walls as well as the width of the ring's potential. Increasing $q$ value, the ring becomes narrower and concurrently outer walls get steeper. For small $q$ values of order 1 and less it distinctly increases the width of the ring's core. The last mentioned parameter $b$ is responsible for height of the ring's core. While increasing $b$ value we make lower the core of the ring.

The appropriate choice of parameters allows us to modify the shape of the potential in the wide range. For example, if we fix exponential parameters $p=0$ and $q=1$, then we obtain the potential in the form of the linear combination of Gaussian functions that was used in Ref. [24]. If we fix the exponential parameter $p=0$ and parameter $b=0$ then we obtain the power-exponential potential which was applied to the description of the realistic quantum dots [25].

The position of the minimum of the potential $V_{\perp}(r)$ corresponds to the average radius of the ring that is denoted by $r_{0}$. This quantity can be found from the extremum condition for $V_{\perp}(r)$ when all above-mentioned parameters are fixed. The number of free parameters can be reduced if we expand the lateral potential 
into the Taylor series around the average radius $r_{0}$, to the second order. This approximation means that for $r$ close to $r_{0}$, the confined potential is described by the displaced parabola, e.g. [14],

$$
V_{\perp}(r) \approx \frac{1}{2} m^{*} \omega_{0}^{2}\left(r-r_{0}\right)^{2}
$$

where $m^{*} \omega_{0}^{2}$ corresponds to the second derivative of potential $V_{\perp}(r)$ at $r_{0}$. The parabolicity of potential at the neighborhood of the average radius of the ring is observed in experiment [3]. The form of confinement potential given by Eq. (1) allows us to use a total wave function in the separated form, namely

$$
\Psi(r, \varphi, z)=\Phi(r, \varphi) \zeta(z)
$$

where the component $\zeta(z)$ of the total wave function is the solution of the Schrödinger equation for the potential $V_{\|}(z)$. The application of the finite well potential instead of the infinity one along the growth axis allows us to simply extend the presented model to a three-dimensional case as it has been considered in Refs. $[15,16]$. We assume that the electronic wave function $\zeta_{n_{z}}(z)$ is frozen to the ground state in the vertical direction. It is a realistic assumption because, as we mentioned before, the quantum rings are not high in the vertical direction. It implies the large separation between energy levels $\Delta E_{\|}=E_{\|}^{\left(n_{z}+1\right)}-E_{\|}^{\left(n_{z}\right)}$ and thus we neglect the transitions to excited states in vertical direction in a first approximation. The function $\Phi(r, \varphi)$ which describes the motion of electrons in $x-y$ plane has a form

$$
\Phi_{m_{l}}(r, \varphi)=\frac{1}{\sqrt{2 \pi}} R(r) \mathrm{e}^{\mathrm{i} m_{l} \varphi},
$$

where $m_{l}=0, \pm 1, \ldots$, is the quantum number of the projection of the angular momentum onto the $z$-axis, and function $R(r)$ is the solution of the lateral eigenvalue problem given by the radial Schrödinger equation,

$$
\left[-\frac{\hbar^{2}}{2 m^{*}}\left(\frac{\mathrm{d}^{2}}{\mathrm{~d} r^{2}}+\frac{1}{r} \frac{\mathrm{d}}{\mathrm{d} r}\right)+\frac{\hbar^{2} m_{l}^{2}}{2 m^{*} r^{2}}+V_{\perp}(r)+E_{\|}^{(\mathrm{GS})}\right] R(r)=E_{\perp} R(r),
$$

for electron in the effective mass approximation and with the confinement potential given by Eq. (2). We note here that the lateral potential is modified by the centrifugal term $\hbar^{2} m_{l}^{2} /\left(2 m^{*} r^{2}\right)$.

\section{Calculation results}

We performed our calculations using the GaAs material parameters, i.e. the effective mass of the conduction band $m^{*}=0.067 m_{\mathrm{e}}$, where $m_{\mathrm{e}}$ is the free electron mass. We calculated numerically the energy spectrum $E_{\perp}^{n_{r}}$ and the eigenfunctions $R_{n_{r}}(r)$ of Eq. (6) using the imaginary time step method [26]. In the first step we apply this method to calculate the electron wave function for the ground state. In Fig. $3 \mathrm{a}-\mathrm{d}$ we plotted the radial wave function for the ground state with $m_{l}=0$, $1,2,3$, respectively. The excited-state wave functions were found using the same procedure associated with the simultaneous orthogonalization to the previously found wave functions for the lower energy levels. 

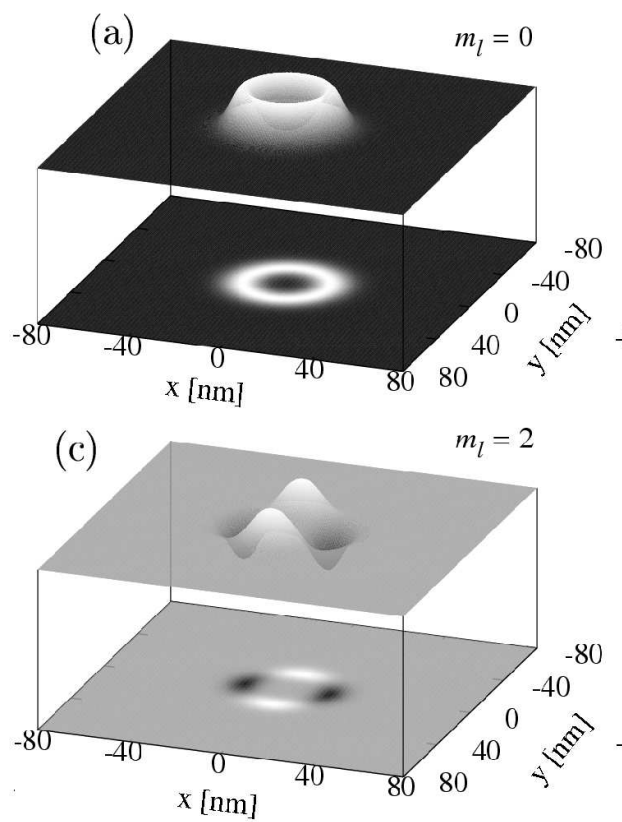

(b)

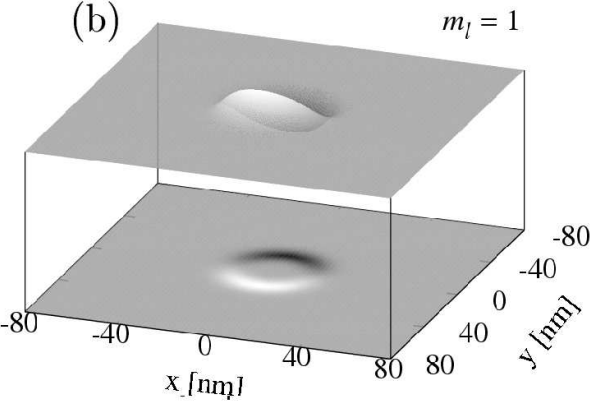

(d)

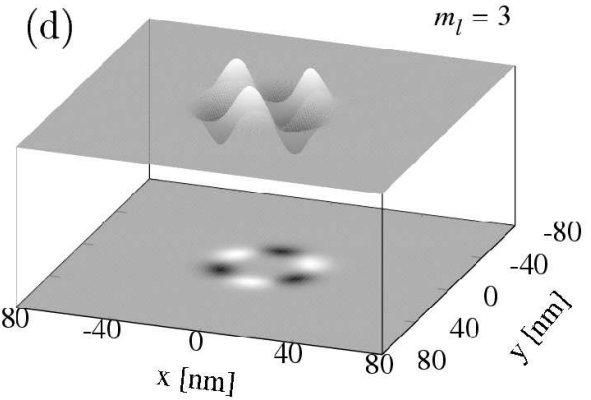

Fig. 3. Two-dimensional electron wave functions for the ground state for $m_{l}=0,1$, 2,3 , respectively. The plot was generated for the following parameters of the potential: $V_{0}=200 \mathrm{meV}, R_{1}=18.5 \mathrm{~nm}, b=0, p=1.0, q=1.0$.

We have calculated the ground state energy as a function of parameters $p, q, R_{1}$, and $b$. The results of these calculations are shown in Fig. 4, and their qualitative explanation needs to include the centrifugal term which changes the shape of repulsive core of the lateral potential. In general, a position of the ground state energy on the energy scale depends on the effective width of quantum ring which is determined by the sum of the lateral potential and the centrifugal one for $m_{l}>0$. In particular, we observe a significant influence of the centrifugal term on the ground state energy for small values of $p$ and $R_{1}$ parameters which modify the width of repulsive core (Fig. 4a and c). Given small values of these parameters the repulsive core of the lateral potential is narrow thus the centrifugal term rises the bottom of the potential higher and in consequence the energy levels, too. On the other hand, the concurrence between the centrifugal term and lateral potential makes that decreasing height of the repulsive core (increasing $b$ parameter) of the lateral potential changes weakly and almost linearly the energy levels (Fig. 4d). In turn, changes of the $q$ parameter have a small influence on the repulsive core width and thus the ground state energy level is determined by the width of the ring and the inclination of the outer wall. The energy shift due to centrifugal term is the same for all $q$ values in the range (Fig. $4 \mathrm{~b}$ ) and ground state energy changes are determined by the $q$ value. 

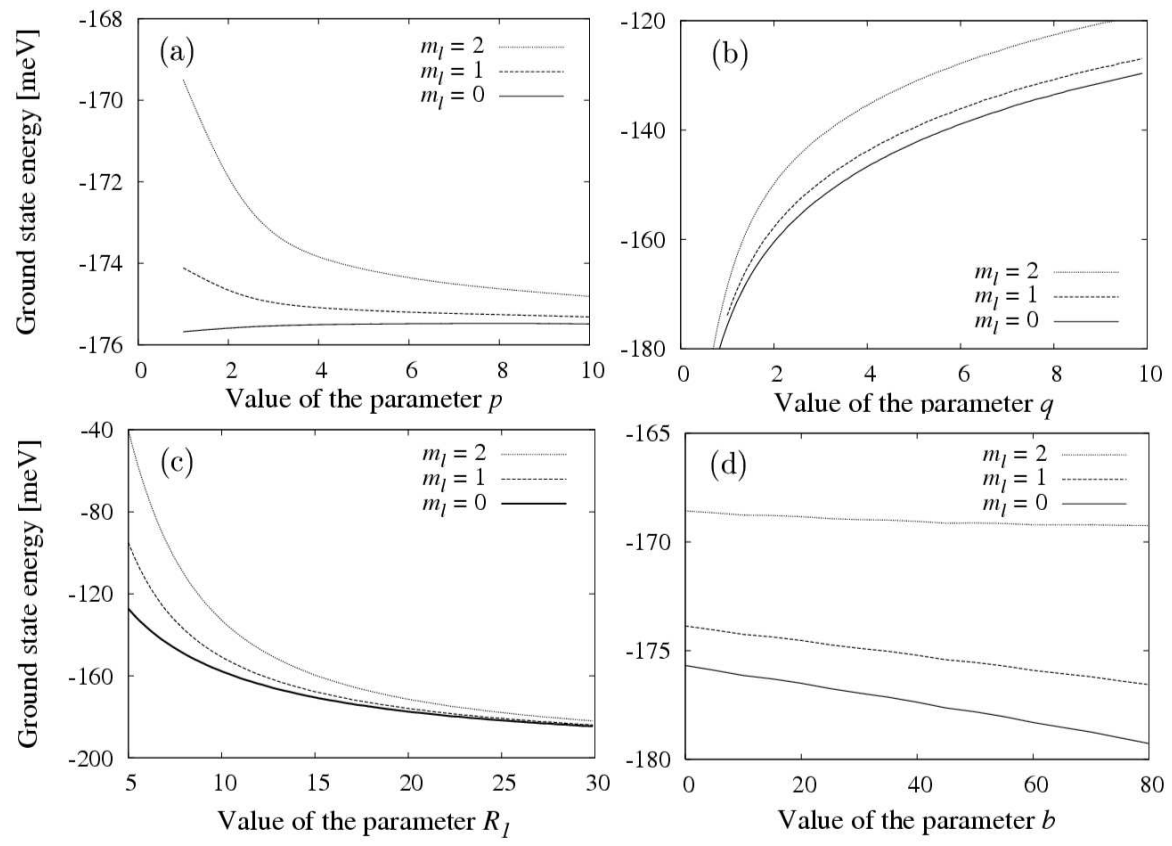

Fig. 4. Ground state energy as a function of the parameter $p(\mathrm{a}), q(\mathrm{~b})$, parameter $R_{1}$ (c) and $b(\mathrm{~d})$ for three consecutive values of the magnetic quantum number; $m_{l}=0-$ solid line, $m_{l}=1$ - dashed line, $m_{l}=2$ - dotted line.

The ground state energy depends on the square of the quantum number $m_{l}$. It means that ground state energy with $m_{l}>0$ is twofold degenerate. The degeneracy is doubled if the spin is taken into account.

\section{Effect of magnetic field}

We now consider the influence of the magnetic field on the electronic properties of quantum ring. The external magnetic field is applied along the growth axis (perpendicular to the ring plane). The total magnetic field consists of the uniform magnetic field $\boldsymbol{B}$ and the flux $\Phi_{\mathrm{AB}}$ through the core of the quantum ring. The vector potential, $\boldsymbol{A}(\boldsymbol{r})$, has a form of the sum of two terms, namely [27-29]:

$$
\boldsymbol{A}(\boldsymbol{r})=\frac{1}{2} \boldsymbol{B} \times \boldsymbol{r}+\frac{\Phi_{\mathrm{AB}}}{2 \pi(\boldsymbol{k} \times \boldsymbol{r})^{2}} \boldsymbol{k} \times \boldsymbol{r}
$$

where $\boldsymbol{k}$ is the versor along the $z$-axis.

The one-band Hamiltonian within the effective mass approximation in cylindrical coordinates including the magnetic field, $\boldsymbol{B}=\nabla \times \boldsymbol{A}(\boldsymbol{r})$, is given by the formula 


$$
\begin{aligned}
\hat{\mathcal{H}}= & -\frac{\hbar}{2 m^{*}} \nabla^{2}+\mathrm{i} \frac{q \hbar}{2 m^{*}}\left(B+\frac{1}{\pi r^{2}} \Phi_{\mathrm{AB}}\right) \frac{\partial}{\partial \varphi}+\frac{q^{2}}{8 m^{*}}\left(B+\frac{1}{\pi r^{2}} \Phi_{\mathrm{AB}}\right)^{2} r^{2} \\
& +g^{*} \frac{q \hbar}{4 m^{*}}\left(\frac{1}{\pi r^{2}} \Phi_{\mathrm{AB}}-B\right) \hat{\sigma}_{z}+V_{\perp}(r)+V_{\|}(z),
\end{aligned}
$$

where $q$ is electronic charge, $\hat{\sigma}_{z}$ denotes $z$ Pauli spin matrix and $\Phi_{\mathrm{AB}}=B \pi d^{2} / 4$ is the magnetic flux through the ring's core. Symbol $d$ denotes the core's diameter that we define as a width in a half-height of the potential $V_{\perp}$. In the present study we neglect the dependence of $g^{*}$-factor on the ring parameters and we use the effective Landé factor for GaAs, i.e. $g^{*}=-0.44$. The magnetic field applied to the system along the $z$-axis does not break the cylindrical symmetry, therefore we use the wave function in the form

$$
\Psi(r, \varphi, z, s)=\frac{1}{\sqrt{2 \pi}} \mathrm{e}^{\mathrm{i} m_{l} \varphi} \zeta(z) R(r) \chi_{s},
$$

where $s$ denotes the spin states, and $\chi_{s}$ represents eigenspinor.

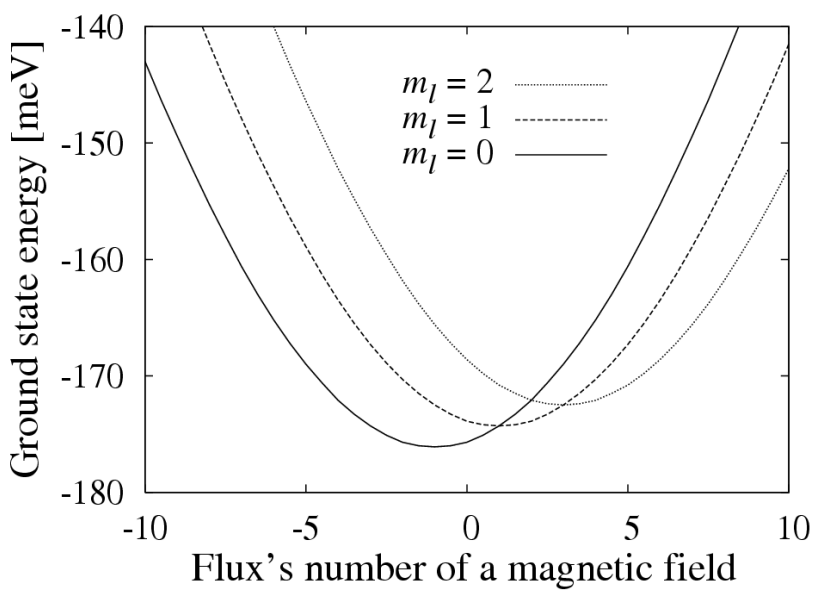

Fig. 5. Ground state energy for a spin-up electron confined in the quantum ring as a function of flux's number of a magnetic field for $m_{l}=0-$ solid line, $m_{l}=1$ - dashed line, $m_{l}=2-$ dotted line.

The form of wave function allows us to reduce the eigenvalue problem for the Schrödinger equation, $\hat{\mathcal{H}} \Psi(r, \varphi, z, s)=E \Psi(r, \varphi, z, s)$, with the Hamiltonian given by Eq. (8) to the eigenvalue problem for the radial Schrödinger equations for each spin state, separately. The energy spectrum of the radial Schrödinger equation is calculated numerically by employing the imaginary-time step method. Figure 5 presents results of our calculation for the ground state energy as a function of flux's number of a magnetic field penetrating the core area.

The calculations were done for the potential $V_{\perp}(r)$ with the following parameters: $V_{0}=200 \mathrm{meV}, R_{1}=18.5 \mathrm{~nm}, b=0, p=1.0, q=1.0$. In the case 
of narrow quantum rings that are modelled by the one-dimensional models, the ground state energy as a function of flux's number is described by the Fock-Darwin curves $E_{0}\left(\Phi_{\mathrm{AB}} / \phi_{0}\right)$ with a minimum always at the same level. In Fig. 4, we see that the minimum of each curve shifts to a larger value of energy for consecutive values of the quantum number $m_{l}>0$ as the flux's number increases. The effect is due to the finite width of the quantum rings [30].

One of the purely quantum effects that occur in the quantum rings is the persistent current. The existence of non-zero persistent current in such systems results from the fact that the electronic wave function is coherent in the presence of magnetic field around the ring. This nontrivial effect is presented in the closed loop structures, if the phase coherent length $\ell_{\varphi}$ is bigger than the ring circumference $2 \pi r_{0}$, where $r_{0}$ is the average radius of the ring [31]. The first measurements of the persistent current in single, isolated metallic loops have been made by Chandrasekhar et al. [20]. The experimental results allowed them to estimate the magnitude of the persistent current of order $(0.3-2.0) I_{0}$, where $I_{0}=e v_{\mathrm{F}} /\left(2 \pi r_{0}\right) \approx$ $5 \mathrm{nA}$ is the theoretical value of the amplitude of the persistent currents [18]. Two years later, Mailly et al. [21] reported the measurements of the persistent current in a semiconductor mesoscopic ring. The authors found a typical current amplitude of $I_{\text {typ }}=\left\langle I^{2}\right\rangle=4 \pm 2 \mathrm{nA}$ after averaging different measurements.

For the quantum rings with a finite width, the amplitude of the persistent current can be estimated by a formula $I=\beta I_{0}$ at $T=0 \mathrm{~K}$, where correction factor $\beta$ is related to the geometrical parameters of the potential and it is proportional to the square root of the transverse channels number $[32,33]$. We calculate the persistent current associated with the single-particle state by integrating the corresponding density of probability current,

$$
j_{n m_{l}}(r)=\left(\frac{m_{l} \hbar}{m^{*} r}-\frac{e \Phi}{2 m^{*} \pi r}-\frac{e B r}{2 m^{*}}\right) R_{n}^{2}(r)
$$

along the radial direction as follows [29]:

$$
I_{n m_{l}}=-e \int \mathrm{d} r j_{n m_{l}}(r)
$$

where $-e$ is the electron charge.

Using these methods of calculation of the persistent currents we include the correction factor $\beta$ in a quite natural way. The total persistent current, $I$, of the ring is given by the formula

$$
I=\sum_{n, m_{l}} I_{n m_{l}}
$$

where the summation runs over all occupied states.

We have calculated the persistent current as a function of flux for different values of parameters $p, q, R_{1}$, and $b$. The results of our calculations are presented in Fig. 6. We observe characteristic oscillations of the persistent current values with the flux's number of a magnetic field as it was presented in $[18,19]$. In our system the period of oscillations is equal to $1\left[\Phi_{\mathrm{AB}} / \phi_{0}\right]$. Furthermore, the period 

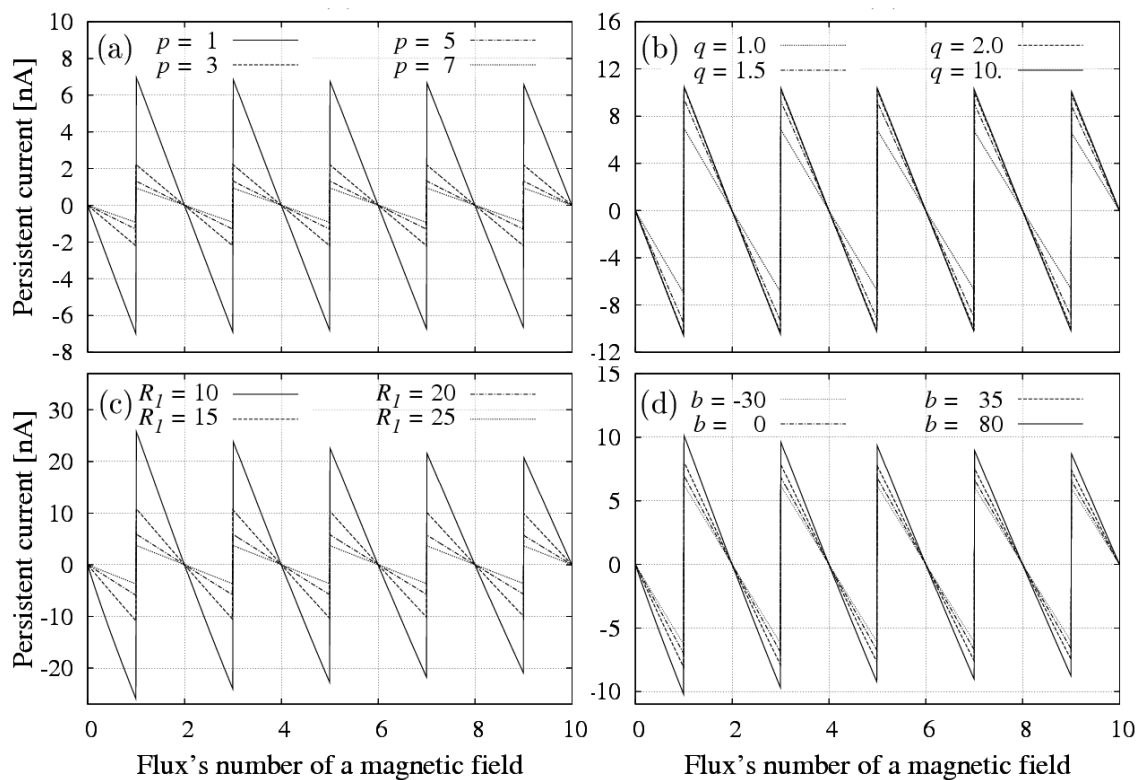

Fig. 6. The persistent current in the ring as a function of the flux $\left(\Phi_{\mathrm{AB}} / \phi_{0}\right)$ for different values of parameters $p(\mathrm{a}), q(\mathrm{~b}), R_{1}(\mathrm{c})$, and $b(\mathrm{~d})$.

is independent of the potential parameters (profile of the potential). The value of the persistent current for a given flux is proportional to parameters $q$ and $b$ and inversely proportional to $p$ and $R_{1}$. In other words, the narrower is the ring and the ring's core the bigger is persistent current value for a given flux. The parameter $p$ is responsible for the width of the ring's core. Increasing $p$ value we broaden the core. The ring average radius grows up moving electron further from the ring's center. In effect, the persistent current amplitude decreases (Fig. 6a). While changing $q$ value we manipulate the width of the ring. When $q$ rises the outer wall of the ring becomes steeper and the width of the ring becomes smaller. Thus the electron is squeezed in a lateral direction and moved toward the ring's core. In consequence, it reacts stronger to magnetic flux changes and the persistent current amplitude grows (Fig. 6b). When $R_{1}$ raises the ring becomes wider and the influence on persistent current (Fig. 6c) is opposite to the effect of changes of $q$ parameter. Changing the parameter $b$ we modify the height of the core. For lower core we achieve a bigger amplitude of the persistent current because the electron can penetrate also the core's area of the ring. In consequence, it interacts stronger with a magnetic field flux penetrating the core's area. For all cases it can be seen that the amplitude of changes of the persistent current slightly decreases as the values of parameters $p, q, R_{1}$, and $b$ increase. This effect originates from increasing flux that influences the width of the repulsive core and moves the electron more outside. 


\section{Concluding remarks}

We have used a two-dimensional confinement potential of a quantum ring to study the effects of shape on its electronic properties. We have performed numerical calculations of the one-electron energy spectrum of two-dimensional quantum rings as a function of the geometric-confinement parameters using the imaginary-time step method. We have shown that ground state energy of the confinement potential is a monotonic function of these parameters. The flexibility of the model of confinement potential allows us to state that it is a very good candidate for the modelling of three-dimensional self-organized quantum rings.

Using this model, we have studied the effects of an external magnetic field (applied in the axial direction of the ring) on the energy spectrum. We also have analyzed the influence of geometric-confinement parameters on the persistent currents in the ballistic regime. We show that the amplitude of the persistent currents is very sensitive to these parameters. The main conclusion of these calculations is that the amplitude of the persistent current's changes is proportional to the core's width and inversely proportional to the ring's width. In other words, the narrower is the ring and its core the larger is amplitude of the persistent current.

In view of increasing interest on fabrication of semiconductor quantum rings with complex shapes using the self-organized techniques, the presented work can be regarded as a basis for the understanding of self-organized semiconductor quantum rings.

\section{Acknowledgments}

This paper was partly supported by the Polish Ministry of Scientific Research and Information Technology in the framework of the solicited grant PBZ-MIN008/P03/2003.

\section{References}

[1] A. Lorke, R.J. Luyken, Physica B 256, 4244 (1998).

[2] H. Pettersson, R.J. Warburton, A. Lorke, K. Karrai, J.P. Kotthaus, J.M. Garcia, P.M. Petroff, Physica E 6, 510 (2000).

[3] A. Lorke, J. Luyken, A.O. Govorov, J.P. Kotthaus, Phys. Rev. Lett. 84, 2223 (2000).

[4] T. Heinzel, R. Held, S. Luscher, K. Ensslin, W. Wegscheider, M. Bichler, Physica E 9, 84 (2001).

[5] T. Raz, D. Ritter, G. Bahir, Appl. Phys. Lett. 82, 1706 (2003).

[6] T. Mano, T. Kuroda, S. Sanguinetti, T. Ochiai, T. Tateno, J. Kim, T. Noda, M. Kawabe, K. Sakoda, G. Kido, N. Koguchi, Nano Lett. 5, 425 (2005).

[7] T. Chakraborty, P. Pietiläinen, Phys. Rev. B 50, 8460 (1994).

[8] A. Emperador, M. Pi, M. Barranco, A. Lorke, Phys. Rev. B 62, 4573 (2000).

[9] S.-S. Li, J.-B. Xia, Appl. Phys. Lett. 89, 3434 (2003). 
[10] D. Gridin, A.T.I. Adamou, R.V. Craster, Phys. Rev. B 69, 155317 (2004).

[11] S. Viefers, P. Koskinen, S.P. Deo, M. Manninen, Physica E 21, 1 (2004).

[12] W.-C. Tan, J.C. Inkson, Semicond. Sci. Technol. 11, 1635 (1996).

[13] Z. Barticevic, M. Pacheco, A. Latgé, Phys. Rev. B 62, 6963 (2000).

[14] J. Simonin, C.R. Proetto, Z. Barticevic, G. Fuster, Phys. Rev. B 70, 205305 (2004).

[15] J. Planelles, W. Jaskólski, J.I. Aliaga, Phys. Rev. B 65, 033306 (2002).

[16] O. Voskoboynikov, Y. Li, H.-M. Lu, C.-F. Shih, C.P. Lee, Phys. Rev. B 66, 155306 (2002).

[17] V. Halonen, P. Pietiläinen, T. Chakraborty, Europhys. Lett. 33, 377 (1996).

[18] N. Byers, C.N. Yang, Phys. Rev. Lett. 7, 46 (1961).

[19] M. Büttiker, Y. Imre, R. Landauer, Phys. Lett. A 96, 365 (1985).

[20] V. Chandrasekhar, R.A. Webb, M.J. Brady, M.B. Ketchen, W.J. Gallagher, A. Kleinsasser, Phys. Rev. Lett. 67, 3578 (1991).

[21] D. Mailly, C. Chapelier, A. Benoit, Phys. Rev. Lett. 70, 2020 (1993).

[22] W. Rabaud, L. Saminadayar, D. Mailly, K. Hasselbach, A. Benoit, B. Etienne, Phys. Rev. Lett. 86, 3124 (2001).

[23] E. Zipper, M. Kurpas, M. Szelag, J. Dajka, M. Szopa, Phys. Rev. B 74, 125426 (2006).

[24] B. Szafran, J. Adamowski, S. Bednarek, J. Phys., Condens. Matter 14, 73 (2002).

[25] M. Ciurla, J. Adamowski, B. Szafran, S. Bednarek, Physica E 15, 261 (2002).

[26] K.T.R. Davies, H. Flocard, S. Krieger, M.S. Weiss, Nucl. Phys. A 342, 111 (1980).

[27] E. Merzbacher, Quantum Mechanics, Wiley, Int. Edition, New York 1998.

[28] E.N. Bogachek, U. Landman, Phys. Rev. B 52, 4067 (1995).

[29] Y. Avishai, Y. Hatsugai, M. Kohomoto, Phys. Rev. B 47, 9501 (1993).

[30] S.M. Reimann, M. Maninnen, Rev. Mod. Phys. 74, 1283 (2002).

[31] U. Eckern, P.J. Schwab, Low Temp. Phys. 126, 1291 (2002).

[32] H.-F. Cheung, Y. Gefen, E.K. Riedel, W.-H. Shih, Phys. Rev. B 37, 6050 (1988).

[33] H.-F. Cheung, E.K. Riedel, Y. Gefen, Phys. Rev. Lett. 62, 587 (1989). 